\title{
A Graph-Theory Algorithm for WCET Estimation
}

\author{
Guowei $\mathrm{Wu}^{1}$ and Kai $\mathrm{Yao}^{2}$ \\ ${ }^{1}$ Software College of Dalian University of Technology, Dalian 116023,China \\ wgwdut adlut.edu.cn \\ ${ }^{2}$ Library Information Center of Shenyang University of Technology, Shenyang \\ 150001, China \\ frantodd2002@yahoo.com.cn
}

\begin{abstract}
The complexity of modern program increases the difficulty to compute the upper bound worst case execution time fast and accurately. A new estimation method for WCET estimation is presented that uses graph theory to solve the program complexity problem. In this method, program basic blocks are represented as vertices in an undirected graph. The resulting graph can be partitioned into connected subgraphs with no edges between them. These subgraphs can in turn be broken into biconnected components. The combinational problem is reduced to finding the maximum energy of these small biconnected components and combining the results to identify the global maximum energy information, that is the worst case execution time. This algorithm will allow to analyze more complex program and can makes the execution time upper bound more accurate.
\end{abstract}

\section{Introduction}

The worst-case execution time (WCET) estimation of a program remains a difficult challenge. Software for today's large embedded real time system is very complex, consisting of many intercommunicating components for various devices and control functions. The field of execution time analysis is both broad and deep. There are many complimentary techniques that can be used to calculate the value of the WCET for a complex system. However each technique assume that there is no interaction between components. To deal with this intractable problem, the researchers trade off prediction accuracy for computational complexity by proposing different pessimistic heuristics, which results in fast but less accurate analysis. Different pessimistic methods are thus proposed to cope with this complexity[1],[2],[3],[4],[5], and they result in loose estimated WCET. Another severe drawback is that they cannot handle any user annotations describing infeasible program paths, which are essential in tightening the estimated WCET. In this article, we will present a new algorithm based on graph theory, which enable to deal with programs that have interaction. A graph $\mathrm{G}=[\mathrm{V}, \mathrm{E}]$ consists of an ordered pair, where $\mathrm{V}$ represents a nonempty set of vertices and $\mathrm{E}$ symbolizes a set of edges. When $\mathrm{V}$ represents the program basic block and $\mathrm{E}$ represents the set of program flows, graph $\mathrm{G}$ becomes a program control flow graph. 
The rest of the paper arranges as follows. Section 2 describe the proposed estimation method, section 3 gives the experiment results, the conclusions and future work are presented in section 4.

\section{Graph-Theory Algorithm for WCET Estimation}

Given that each instruction takes a constant time to execute, the total execution time can be computed by summing the product of instruction counts by their corresponding instruction execution times. Since all instructions within a basic block must have the same execution counts, they can be considered as a single unit. If we let $x_{i}$ be the execution count of a basic block $M_{i}$ and $c_{i}$ be the execution time of the basic block, then given that there are $N$ basic blocks, the total execution time of the program is given as:

$$
T=\sum_{i}^{N} c_{i} x_{i}
$$

The possible values of $x_{i}$ are constrained by the program structure and the possible values of the program variables. If we can represent these constraints as linear inequalities, then the problem of finding the estimated WCET of a program will become an ILP problem.

The linear constraints can be divided into two parts: program structural constraints, which are derived from the program's control flow graph (CFG), and program functionality constraints, which are provided by the user to specify loop bounds and other path information.

The accuracy of program control flow information effect the accuracy of WCET estimation. In our algorithm, we can represent the program basic block in terms of graph theory by making each residue a vertex in an undirected graph. If at least one basic block of residue $i$ interacts with at least one basic block of residue $j$, then there is an edge between vertices $i$ and $j$ of the graph. The active residues can be represented as a graph, the active residues can therefore be grouped into interacting clusters. Residues in different clusters do not have contacts with one another. Each cluster is a connected subgraph of the entire graph. The problem is therefore to enumerating the combinations of the basic block for the residues in each connected graph. Assuming each residues has the same number of basic block $n_{b l k}$. The graph in Figure 1 has one residue labeled as a dark gray vertex that breaks the graph into two pieces when removed. The global maximum of the execution time can be found by identifying the maximum execution time configuration for each subgraph once for each basic block of the keystone residues. The solution is the one that finds the maximum execution time using equation (2), where EL is the largest execution time combination of basic block in the left subgraph, ER is the right subgraph, Eself is the execution time of interaction of the basic blocks. According to different programs, give the constraints, all the constraints are passed with the goal of maximizing cost function (2). The solver will return the estimated WCET. 
To improve the process speed, we break up clusters of interacting basic block into

$$
E=\max _{r_{i}}\left\{E_{L}\left(r_{i}\right)+E_{R}\left(r_{i}\right)+E_{\text {self }}\left(r_{i}\right)\right\}
$$

biconnected components of an undirected graph. Biconnected graphs are those that cannot be broken apart by removal of a single vertex. Biconnected graphs are cycles, nested cycles, or a single pair of residues connected by an edge. Vertices that appear in more than one biconnected component are called "repoint". Removing a repoint from a graph breaks the graph into two separate subgraphs. Finding biconnected components and their repoints is easily accomplished by using standard depth-first search algorithm from graph theory contained in many computer science textbooks. For each biconnected component with only one repoint, we find the maximum execution time over all combinations of basic blocks of the residues in the component. This execution time includes all interactions among these residues and between these residues. The worst case execution path is the path that has the most bidirect line path. In Figure 1 , the worst case execution path is $(1,2,3,4,5, \ldots 12)$.

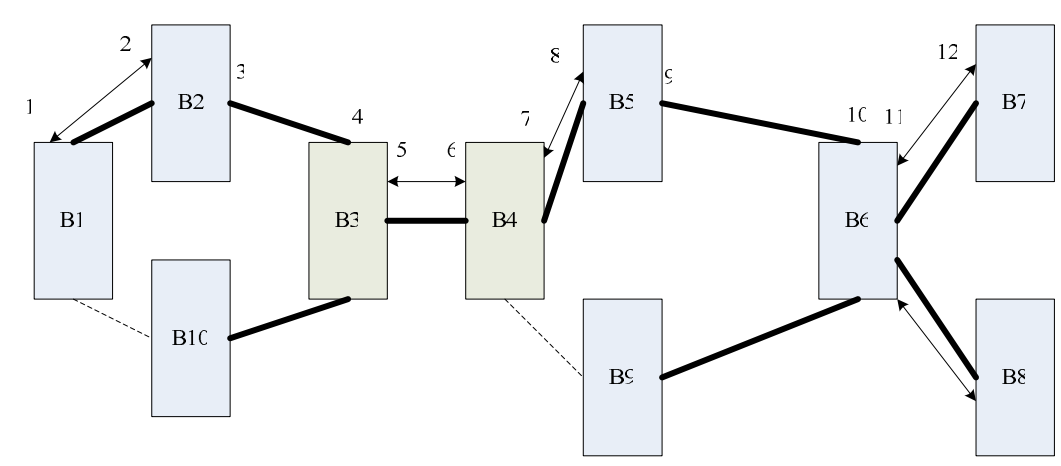

Fig. 1. Flow graph using graph theory for software worst case execution time estimation

\section{Algorithm 1.}

Input: A new program sequence.

Output: Print the fixed basic block and the maximum execution time.

1. Rotamers: for each residue,read in rotamer dihedral angles and probabilities to determine which pairs of basic block may be interact.

2. Disulfides: if desired, determine likely disulfide pairings.Fix the basic block that are designated disulfides for the rest of the calculation.

3. Dead-end elimination: perform a DEE of rotamers that cannot be part of the global maximum execution configuration by using the "Goldstein criterion". The Goldstein criterion is the simplest version of DEE. If the total execution time for all basic blocks is expressed as the sum of self and pairwise execution time, see equation (3). The execution time of interaction of these fixed basic blocks with any unfixed basic blocks is added to the self-execution of the unfixed basic blocks. That is equation (4). 
4. Residue graph: define residues that have more than one rotamer left after the DEE step as "active residues". That is, determine which sets of residues form connected graphs, given the list of edges.

5. Biconnected components:for each cluster, determine the set of biconnected components and repoints in the graph by using a depth-first search procedure. The order of each repoint is defined.

6. Solve clusters: find the maximum execution time for each connected graph(cluster) in turn, for each biconnected component of the cluster with only one repoint,find the maximum execution time of the residues in the component for each rotamer of the repoint each connected graph. A branch-and-bound backtracking algorithm is used for this purpose, in which the residues in the component are sorted from lowest to highest number of rotamers.

$$
\begin{gathered}
E=\sum_{i=1}^{N} E_{\text {self }}\left(r_{i}\right)+\sum_{i=1}^{N-1} \sum_{j>i}^{N} E_{\text {pair }}\left(r_{i}, r_{j}\right) \\
E_{\text {self }}\left(r_{i}\right)=\sum_{i=1}^{N} E_{b b}\left(r_{i}\right)+\sum_{j \in \text { fixed }} E_{\text {pair }}\left(r_{i}, r_{j}\right)
\end{gathered}
$$

The maximum execution time is the maximum execution time of the self residues plus the maximum execution time pairwise of these residues. The bound is defined as:

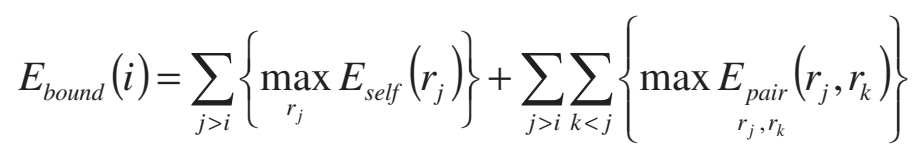

\section{Experimental Results}

To verify the proposed method, we use Motorola MMC2107 development board as target platform which is containing a $40 \mathrm{MHz}$ Motorola MMC2107 processor, $128 \mathrm{~KB}$ of main memory and several I/O peripherals. The M2107 processor contains an onchip 8KB direct-mapped instruction cache organized as 32-16-byte lines.

We select the set of benchmark programs from [1] for our evaluation. Since it is impractical to simulate all the possible program input data and all initial system states, a program's actual WCET cannot be computed. We use the measured WCET in [1] as the actual WCET, We assume that the measured WCET of a program is very close to its actual WCET. Table 1 is the experimental results, time unit is clock period counts, it shows that the proposed method give a more accurate WCET estimation than Wu's method[6]. By using graph theory can extract accurate program flow graph, and thus make the estimation more accurate, and the results prove that the method we give is right and feasible. The result is more accurate than the result in our previous work[6]. So it is an effective approach to use graph theory for embedded real time software WCET estimation. 
Table 1. WCET estimation experimental result

\begin{tabular}{clcc}
\hline Function & $\begin{array}{l}\text { Measured } \\
\text { WECT }\end{array}$ & $\begin{array}{l}\text { Proposed } \\
\text { method }\end{array}$ & Wu's method \\
FFT & $1.25 * 10^{6}$ & $1.21 * 10^{6}$ & $1.18 * 10^{6}$ \\
DES & $2.42 * 10^{5}$ & $2.41 * 10^{5}$ & $2.38 * 10^{5}$ \\
Stats & $1.65 * 10^{4}$ & $1.63 * 10^{4}$ & $1.59 * 10^{4}$ \\
DCT & $1.15^{4} 10^{5}$ & $1.14 * 10^{5}$ & $1.1 * 10^{5}$ \\
\hline
\end{tabular}

\section{Conclusion}

In this paper, we present a new method to find a tight bound on the worst case execution time of real-time software. This approach combines graph-theory to extract program flow graph and can deal with the program that have interaction between components. Experimental results show that the estimated WCET is much closer to the measured WCET. Our next research work will focus on combining data cache and TLB cache analysis into graph-theory algorithm analysis, thus the WCET estimation result will get more accurate.

\section{Acknowledgment}

The authors are gratefully acknowledge support from NSF grants 60673046,and thank Professor Mingchu.Li for providing helpful comments and information.

\section{References}

1. Liu,J.C.,Lee,H.J.:Deterministic Upperbounds of Worst-case Execution Times of Cached Programs. In: Proceeding of the 15th IEEE Read-Time Systems Symposium,Vol.30,New York(1998)182-191.

2. Lim,S.S.,Young,H.B.,Gu,T.J.:An Accurate Worst Case Timing Analysis Technique for RISC Processors. In: Proceeding of the 15th IEEE Real-Time Systems Symposium,Vol.30, New York(1998)97-108.

3. Alan,C.S.:Reasoning about Time in Higher-level Language Software. IEEE Transactions on Software Engineering, Vol.15,No.7,pp.875-889,July 1999.

4. Robert, A.: Bounding Worst-case Instruction Cache Performance. In: Proceeding of the 15th IEEE Real-Time Systems Symposium,Vol.30, New York(1998)172-181.

5. Li,Y.S.,Malik,S., Wolfe, A.: Cache Modeling for Real-Time Software Beyond Direct Mapped Instruction Caches. In: Proceeding of the 17th IEEE Real-Time Systems Symposium, Vol.35, New York (2002)35-42.

6. Wu ,GW.,Yao,lin.: A New WCET Estimation Algorithm based on Instruction Cache and Prefetching Combined Model. Lecture Notes in Computer Science ,Vol (3605). SpringerVerlag, Berlin Heidelberg New York (2005) 557-562. 